\title{
Performance Analysis of Coding Scheme for Wireless Relaying Network
}

\author{
Jing An
}

\author{
Department of Information Engineering, Shijiazhuang Vocational Technology Institute, Shijiazhuang \\ 050081, China \\ anj.sjz@hotmail.com
}

Keywords: Relay; Coding; Wireless communication; Cooperation; Network

\begin{abstract}
This cooperative communication can achieve the additional benefits of cooperative coding in providing diversity and coding gains, and relay has been identified as a fundamental feature in LTE-Advanced in order to achieve LTE-A requirements. In this paper, we introduced a new framework, called hierarchical coding scheme, which can provide different error protections based on different channel situations. Numerical results demonstrate that both cooperation can achieve full diversity and provide significant gains over noncooperation transmission. Hierarchical coding scheme generally performs better than original coded cooperation, especially on FER performance.
\end{abstract}

\section{Introduction}

Relay has been identified as a fundamental feature in LTE-Advanced in order to achieve LTE-A requirements [1]. There are now two types of relays defined in LTE A study: type I relay has its own cell id, and therefore deployed as a separate cell while using donor cell resources for backhauling; type II relay [2-3] doesn't have its own id, and will therefore not introduce any new cell. The cooperative transmission between eNB and relay node $(\mathrm{RN})$ can provide some performance gain under some scenarios. Orthogonal interference-free cooperation can be seen a simple method to fulfill the cooperative transmission without any interference at eNBs. However, the phases of the channel coefficients between eNB to UE and eNB to RN are not the same because of the effects of path-loss, shadowing and slow flat fading [4], it may not guarantee the performance gain by choosing coding scheme based on the inferior link eNB-UE.

In this study, we consider a simple relay scenario shown in Fig.1, where S, R and D denote the source node, RN and destination node. The RN is assumed to be deployed close to the cell edge. In practical scenario, the source node (as well as destination node) can be either an eNB or a UE determined by whether DL transmission or UL transmission. As DL and UL in the forwarding strategies context are similar to each other, we will only focus on the downlink case for convenience, where $\mathrm{S}$ represents eNB and D represents UE.

In this paper, we provide a hierarchical coding scheme at eNB in the cooperative transmission. The information transmitted on the inferior link is provided by a stronger coding and the information transmitted on the superior link is provided by a weaker error protection. Upon receiving the signals from RN and eNB, UE performs a joint Viterbi decoding. This scheme can achieve the additional benefits of cooperative coding in providing diversity and coding gains.

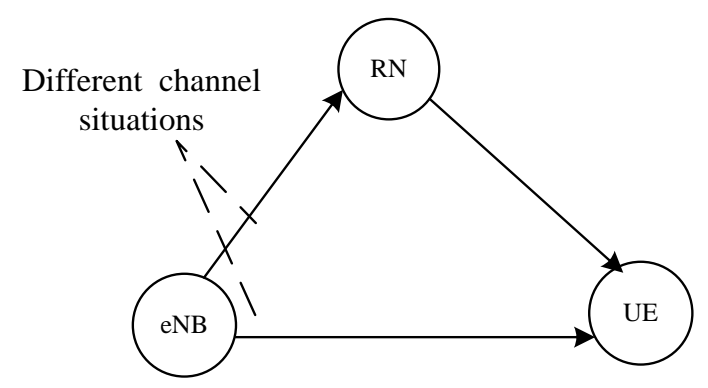

Figure 1. Different channel situations in cooperation 


\section{System Model}

In this section, the performance analysis of amplify-and-forward and decode-and-forward is respectively given from an information theoretic perspective.

AF relays (amplify-and-forward relays). In AF relays, the $\mathrm{RN}$ first amplifies the received signal from UE, and then forwards to UE it serves, as can be seen in Fig. 2, the received signals of the eNB-RN, eNB-UE and RN-UE, respectively, are

$y_{R 1}=h_{S R} x_{s}+n_{R 1}$

$y_{D 1}=h_{S D} x_{s}+n_{D 1}$

$y_{D 2}=h_{R D} \beta y_{R 1}+n_{D 2}$

where $\beta$ is the maximal power amplification factor available at RN; i.e., it always amplifies the received power plus noise to maximum RN transmission power. In this case, the SNR at UE is the sum of the power received directly from eNB and amplified by the RN. And the eNB-RN-UE link SNR at UE can be given by [1]:

$$
S N R=\frac{S N R(S R) * S N R(R D)}{1+S N R(S R)+S N R(R D)}
$$
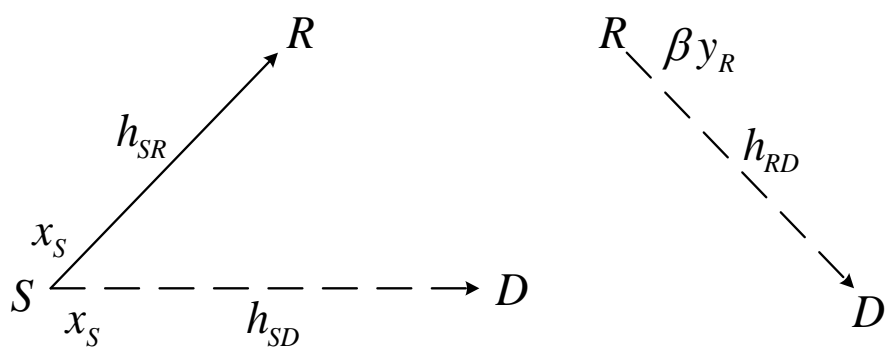

Figure 2. The relay transmission of AF mode

DF relays (decode-and-forward relays). The DF relays is shown in Fig.3, where RN first decodes the signal from eNB, and then transmits it to the UE. The received signals of the eNB-RN, eNB-UE and RN-UE links, respectively.

In this case, the SNR at UE is the sum of the power received directly from eNB and RN. Similarly, the eNB-RN-UE link SNR at UE can be given by [2]:

$$
\mathrm{SNR}=\min \{\mathrm{SNR}(\mathrm{SR}), \mathrm{SNR}(\mathrm{RD})\}
$$
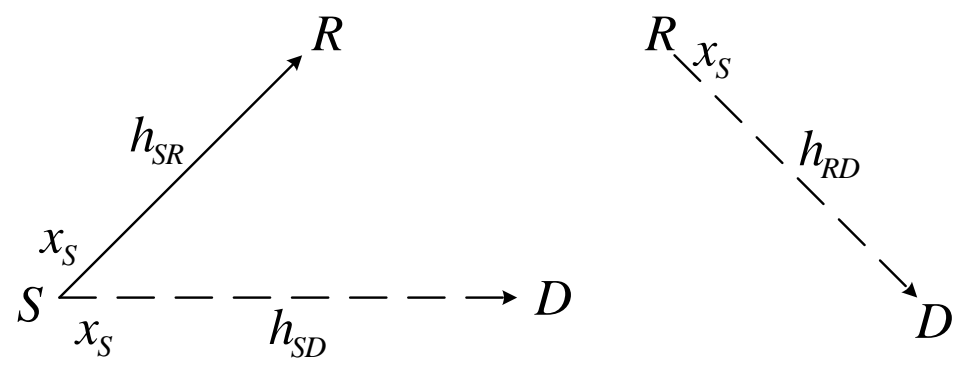

Figure 3. The relay transmission of DF mode

\section{Proposed Coding Scheme}

There are several forwarding strategies for RN, including AF, DF and EF. We focus on DF relaying. We assume that all terminals are equipped with single antenna and cannot transmit and receive simultaneously. All the channels are quasi-static Rayleigh fading. 
Now we begin to introduce the hierarchical coding scheme, whose detailed structure is shown in Fig. $4, \mathbf{m}=\left[m_{1}, m_{2}, \cdots, m_{K}\right] \in\{0,1\}^{K}$ denotes the information bits generated at eNB and the codeword $\mathrm{i}$ $\mathbf{c}=\left[c_{1}, c_{2}, \cdots, c_{N}\right] \in\{0,1\}^{N}, R_{C}=K / N$. Then the coded bits are mapped into modulated signals $\mathbf{x}=\left[x_{1}, x_{2}, \cdots, x_{n}\right], x_{i} \in \mathrm{X}, n=N / \log _{2} M, M=|\mathrm{X}|$ denotes the numbers of constellation points.

According to our scheme, codeword $\mathbf{c}$ is partitioned into two sub-codewords: $\mathbf{c}_{\mathbf{1}}=\left[c_{11}, c_{12}, \cdots, c_{1 N_{1}}\right]$ is the codeword generated by a weaker-protective convolutional encoder with rate $R_{C_{1}}=K / N_{1}$, denoted by Enc1; $\mathbf{c}_{\mathbf{2}}=\left[c_{21}, c_{22}, \cdots, c_{2 N_{2}}\right]$ is the codeword generated by a stronger-protective convolutional encoder with rate $R_{C 2}=K / N_{2}$, denoted by Enc2, $N=N_{1}+N_{2}$. Hence, the modulate signal $\mathbf{x}$ is partitioned into two modulated signals, $\mathbf{x}_{1}=\left[x_{11}, x_{12}, \cdots, x_{1 n_{1}}\right], \mathbf{x}_{2}=\left[x_{21}, x_{22}, \cdots, x_{2 n_{2}}\right]$, and $n_{1}+n_{2}=n$.

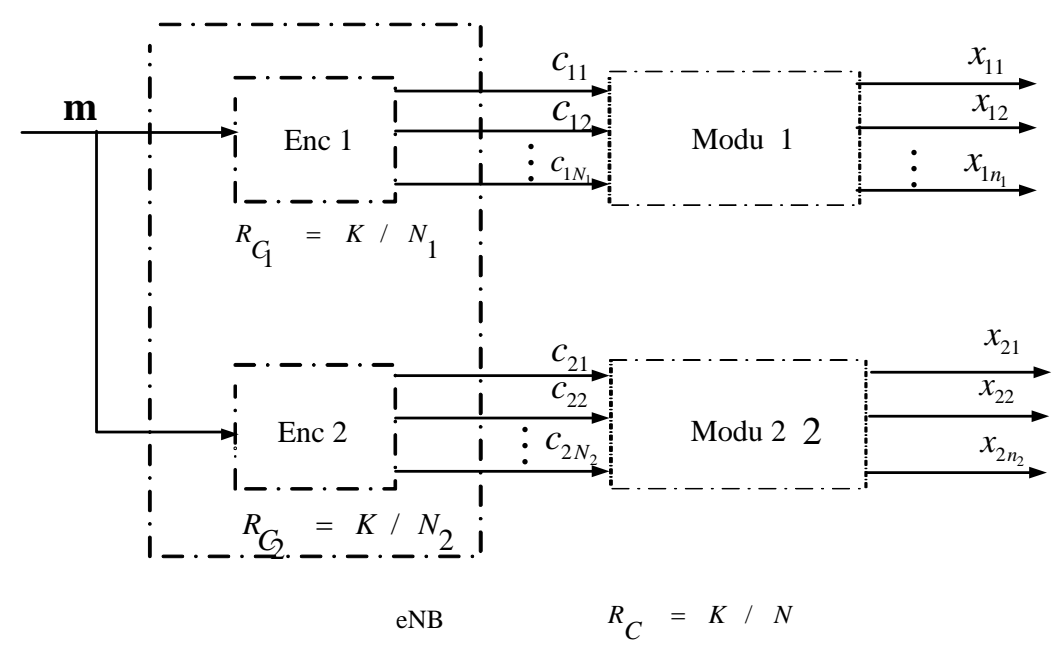

Figure 4. Encoding scheme in eNB

During the transmit frame, eNB broadcasts its information to RN and UE by convolutional Enc1. RN operates in the DF model, so it decodes the information received from eNB firstly, and then re-encodes it with a different convolutional Enc2. If RN cannot decode the information correctly, the system turns into a non-cooperation model automatically. In the cooperative frame, $\mathrm{RN}$ forwards new signal sequence to UE while eNB transmits information too. Upon receiving two versions of the same codewords, UE combines them by using an MRC and the information bits are detected with a Viterbi decoder with the received signal in the first frame.

The effect of channel errors at RN can be categorized into two cases: RN correctly decodes eNB or not. The conditional PEP can be pressed as

$P_{S}\left(d \mid \gamma_{S R}, \gamma_{R D}, \gamma_{S D}\right)=P_{1}\left(d \mid \gamma_{S R}, \gamma_{R D}, \gamma_{S D}\right)+P_{2}\left(d \mid \gamma_{S R}, \gamma_{R D}, \gamma_{S D}\right)$

$P_{1}\left(d \mid \gamma_{S R}, \gamma_{R D}, \gamma_{S D}\right)$ denotes the case that RN can decodes eNB code correctly, and $P_{2}\left(d \mid \gamma_{S R}, \gamma_{R D}, \gamma_{S D}\right)$ denotes the case that RN cannot decode eNB code correctly.

$P_{1}\left(d \mid \gamma_{S R}, \gamma_{R D}, \gamma_{S D}\right)=\left[1-P_{S R}\left(\gamma_{S R}\right)\right] P_{R D}\left(\gamma_{R D}\right) P_{S D}\left(\gamma_{S D}\right)$

$P_{2}\left(d \mid \gamma_{S R}, \gamma_{R D}, \gamma_{S D}\right)=P_{S R}\left(\gamma_{S R}\right) P_{S D}\left(\gamma_{S D}\right)$

where $P_{S R}\left(\gamma_{S R}\right), P_{S D}\left(\gamma_{S D}\right), P_{R D}\left(\gamma_{R D}\right)$ are the conditional PEPs on the corresponding link. $\gamma$ is the instantaneous received SNR, $\gamma=E_{S}|h|^{2} / N_{0}$. Define $\bar{\gamma}$ the average SNR per information bit, (1) can be changed to 


$$
\begin{aligned}
P(d)= & {\left[\pi \bar{\gamma}_{S D}\left(R_{C_{1}} d_{1}+(1-\alpha) R_{C_{2}} d_{2}\right)\right]^{-1} \int_{0}^{(M-1) \pi / M} \sin \theta_{2}^{4} d \theta_{2} \square\left(\alpha R_{C 2} d_{2} \bar{\gamma}_{R D}\right)^{-1} } \\
& -\left[\pi \bar{\gamma}_{S D}\left(R_{C_{1}} d_{1}+(1-\alpha) R_{C_{2}} d_{2}\right)\right]^{-1} \int_{0}^{(M-1) \pi / M} \int_{0}^{(M-1) \pi / M} \sin \theta_{1}^{4} \sin \theta_{2}^{2} d \theta_{1} \theta_{2}\left(\alpha R_{C 2} d_{2} \bar{\gamma}_{R D}{ }^{-1} \pi R_{C_{1}} d_{1} \bar{\gamma}_{S R}\right)^{-1} \\
& +\left[\pi \bar{\gamma}_{S D}\left(R_{C_{1}} d_{1}+(1-\alpha) R_{C_{2}} d_{2}\right)\right]^{-1} \int_{0}^{(M-1) \pi / M} \int_{0}^{(M-1) \pi / M} \sin \theta_{1}^{2} \sin \theta_{2}^{2} d \theta_{1} d \theta_{2} \square\left(\pi R_{C_{1}} d_{1} \bar{\gamma}_{S R}\right)^{-1}
\end{aligned}
$$

where $0 \leq \alpha \leq 1$ is a ratio to maintain the same average power in the cooperative frame, $d_{1}$ and $d_{2}$ are the numbers of bits in the Hamming weight in two frames, and $d_{1}+d_{2}=d$. It can be seen from $\mathrm{Eq}(4)$ that system performance is correlate with the first part, which is inverse proportion to $d_{2}$. Therefore, reducing $d_{1}$ by providing a weaker error protection in the transmit frame and increasing $d_{2}$ by providing a stronger error protection in the cooperative frame can improve system performance effectively without increasing complexity.

\section{Simulation Results}

In order to evaluate the performance of hierarchical coding cooperation scheme (HCC), we use a convolutional encoder with generator polynomials $(5,7,15,17)$ in octal notation, which can provide two different protections based on its different constituent encoders. For simplicity, BPSK modulation is assumed. All the channels are assumed to be independent quasi-static fading channel where the channel coefficients are fixed for the duration of the frame. The transmitted frame size in each slot is equal to 130 coded bits. The original coded cooperation (OCC) transmission is illustrated for comparison in which two encoders with the same protection are used.

It is clearly shown in Fig. 5, when $\gamma_{S R}=\gamma_{R D}$, HCC can provide a gain of about $2.5 \mathrm{~dB}$ when the average SNR of eNB-RN is $30 \mathrm{~dB}$, while a little improvement provided at $20 \mathrm{~dB}$ compare with OCC. When $\gamma_{S R}>\gamma_{R D}$, HCC can obtain a coding gain of about $1 \mathrm{~dB}$; when $\gamma_{S R}<\gamma_{R D}$, the coding gain can improve to $1.5 \mathrm{~dB}$. Again we see that, even when UE is far away from eNB, our proposed scheme can achieve the additional benefits of cooperative coding in providing diversity and coding gains at any different channels.

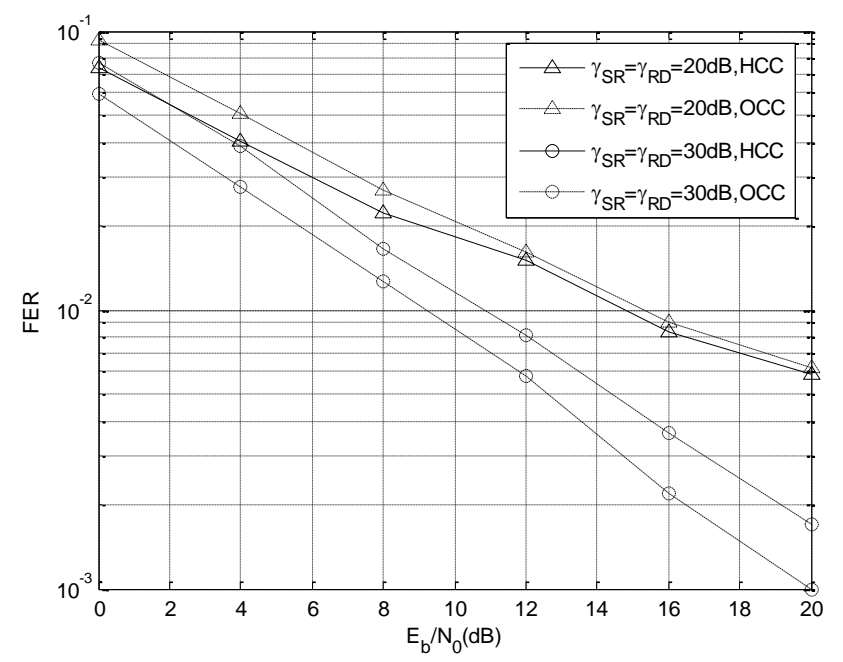

Figure 5. $\gamma_{S R}=\gamma_{R D}$ FER Performance for different strategies

\section{Conclusion}

In this paper, we have introduced a new framework, called hierarchical coding scheme, which can provide different error protections based on different channel situations. Numerical results demonstrate that both cooperation can achieve full diversity and provide significant gains over 
noncooperation transmission. Hierarchical coding scheme generally performs better than original coded cooperation, especially on FER performance. The proposed strategy can be extended to the joint design of code and modulation on the asymmetric wireless channel.

\section{References}

[1] P. A. Anghel and M. Kaveh, "Exact symbol error probability of a cooperative network in a Rayleigh-fading environment", IEEE Trans. on Wireless Communications, vol. 3, Sep. 2004, pp.1-5.

[2] T. Wang, A. Cano, J. N. Laneman, "High-Performance Cooperative Demodulation With Decode-and-Forward Relays", IEEE Trans. on Communications, Vol. 55, July 2007, pp.1-5.

[3] R1-091763, Cell Edge Performance for Amplify and Forward vs. Decode and Forward Relays, Nokia Siemens Networks, Nokia

[4] R1-090880, Analysis of decode and forward relaying strategies, ETRI

[5] Li Chang, Wang Yafeng, Xiang Wei and Yang Dacheng, "Outage Probability Analysis of Coded Cooperation with Multiple Relays," IEEE VTC 2011 Fall, San Francisco, United States, Sept. 5-8, pp. 1-5.

[6] P.K. Sharma, P. Garg, "Outage Analysis of Coded Cooperation with Multiple relays and Nakagami-m Fading," in Proc.of IEEE Communications Workshops (ICC), Budapest, Hungary, 2013, pp. 322 - 326.

[7] P.K.Sharma, and P.Garg, "Coded Cooperation: Generalised Outage Analysis," IET Communications, vol.8, pp. 972 - 979, Jun. 2014.

[8] T.X.Vu, P.Duhamel, and M.Di Renzo, "Performance Analysis of Network Coded Cooperation with Channel Coding and Adaptive DF-Based Relaying in Rayleigh Fading Channels," IEEE Signal Processing Letters, vol.22, pp. 1354 - 1358, Sept. 2015.

[9] A.R. Heidarpour, G.K. Kurt, M. Uysal, "Diversity-multiplexing tradeoff for network coded cooperative OFDMA systems," in Proc. IEEE International Conference on Communications (ICC), London, 2015, pp. 4368 - 4373.

[10]K. Ishii, K. Ishibashi, and H. Ochiai, "Multilevel Coded Cooperation for Multiple Sources," IEEE Transactions on Wireless Communications, vol. 10, pp. 4258-4269, Dec. 2011.

[11]Hausl. C, Lutz. T, Koetter, "Distributed turbo coding with information transfer via timing of the half-duplex relay-phases," Proc. IEEE ICC 2009, May 2009, pp. 1-5.

[12]Y. Li, B. Vucetic and J. Yuan, "Distributed turbo coding with hybrid relaying protocols," Proc. IEEE PIMRC 2008, Sept. 2008, pp.1-5. 